\title{
Evaluación del impacto de los programas PCPI del Servicio de Empleo de Catalunya
}

Núm. 2 (2014), pp. 187-223.

Jaume Blasco², David Casado ${ }^{3}$, Jordi Sanz ${ }^{4}$ y Federico A. Todeschini 5

Recibido: Enero, 2014

Aceptado: Marzo, 2014

JEL Clasif: JEL-class: J64, J68, J13, I28.

\footnotetext{
${ }^{1}$ Este trabajo ha sido elaborado dentro de la evaluación de las políticas activas de ocupación del Servicio de Ocupación de Catalunya co-financiado por el Departament d'Empresa i Ocupació de la Generalitat de Catalunya y el Instituto Catalán de Evaluación de Políticas Públicas (Ivàlua). Los autores agradecen los comentarios de Kevin Hollembeck, Miquel Àngel Alegre y de Sergi Jiménez. Cualquier error es responsabilidad única de los autores. Los informes completos pueden accederse en www.ivalua.cat. Correspondencia: Federico.Todeschini@ivalua.cat

22Ivalua, jaume.blasco@nyu.edu

3 Ivalua, david.casado@ivalua.cat

4 Ivalua, jordi.sanz@ivalua.cat

5 Ivalua federico.todeschini@ivalua.cat
} 


\begin{abstract}
In this paper we perform an impact evaluation of the PCPI program, a public sponsored training program for the youth of the Catalonian Employment Service. This program, in its different variants, has the double objective of integrating participants into the labor market as well as re-engaging them back into the formal education system. Using a unique and rich database that combines administrative records from the unemployment office and the social security we apply the matching technique, in order to compare outcomes of participants and and non-participants unemployed individuals with similar observable characteristics. We are able to follow the labor and education outcomes for 3 years after the end of the program. We obtain that none of the variants of the PCPI program has a statistically significant labor impact in the medium term, although they do have a 'lock in' effect in the short term. However, both variants of the program are quite effective in reengaging participants back into formal education, although the reengagement is not translated into higher graduation rates.
\end{abstract}

Key Words: dropouts, youth unemployment, active labor market policies, propensity score matching

\title{
Resumen
}

En el presente trabajo evaluamos el impacto de dos variantes del programa PCPI del Servicio de Empleo de Catalunya (SOC). Estos programas tienen un doble objetivo: por un lado buscan posibilitar a los participantes una inserción sociolaboral satisfactoria, y por el otro buscan ampliarles sus competencias básicas para permitirles proseguir sus estudios. A partir de una base de datos muy completa, combinando información proveniente de los registros del SOC y de la Seguridad Social, aplicamos la técnica matching, para comparar participantes en cada programa con desempleados no participantes de características observables similares, y seguimos su evolución laboral y educativa durante 3 años después de acabado el programa. De acuerdo a los resultados, ninguna de las dos políticas activas de empleo analizadas tiene un impacto laboral significativo en el mediano plazo, aunque sí tienen consecuencias negativas en el corto plazo en forma de efecto 'lock-in'. Sin embargo, los dos programas tienen una efectividad muy elevada para conseguir un retorno a la educación reglada, sea en la ESO o en Ciclos Formativos de Grado Medio. No obstante ello, las tasas de graduación posterior son relativamente bajas. 
Evaluación del impacto de los programas PCPI del Servicio de Empleo de Catalunya

Palabras clave: abandono escolar, desempleo juvenil, políticas activas de empleo, propensity score matching 


\section{Introducción}

La actual crisis económica está golpeando con especial dureza a los jóvenes. De forma similar al resto de países de nuestro entorno, la tasa de desempleo juvenil duplica la tasa de desempleo de la población adulta, con el agravante de que los niveles de desempleo de España y Cataluña se encuentran entre los más altos de la Unión Europea. En particular, según las cifras de la Encuesta de población activa (EPA) del segundo trimestre de 2013, la tasa de desempleo en Cataluña de los jóvenes de entre 16 y 24 años se situaba en el 53,8 \% mientras que la de la población de entre 25 y 54 años era del 20,8 \%. Dentro del colectivo juvenil, la situación resulta aún más dramática para los jóvenes que abandonaron prematuramente el sistema educativo habiendo obtenido, como máximo, el título de la ESO, que en el caso de Cataluña representaban en el año 2012 un 24,9 \% de la población de entre 18 y 24 años, uno de los peores registros entre los países de nuestro entorno (UE27: 12,8 \%). Así, con la brutal contracción de la oferta de lugares de trabajo de baja cualificación, los jóvenes con un bajo nivel formativo han visto desvanecerse completamente sus posibilidades de inserción laboral.

Reconducir los problemas anteriores es una tarea compleja y requiere actuar sobre diferentes frentes y con horizontes temporales diversos: por un lado, para conseguir mejoras a más largo plazo, parece prioritario intentar reducir los índices actuales de fracaso escolar y, en el ámbito laboral, acabar con un mercado dual en el que los jóvenes se llevan la peor parte, especialmente en tiempos de crisis. Por otra parte, mientras no se avance en estos aspectos, es evidente que hay que intentar mejorar, ahora y aquí, las perspectivas laborales de los jóvenes que actualmente se encuentran desempleados.

En este último sentido, el Servei d'Ocupació de Catalunya (SOC) impulsa, en el marco de su oferta de políticas activas, un conjunto de programas dirigidos a jóvenes desempleados que, debido a su bajo nivel formativo, tienen muchas dificultades para acceder al mercado de trabajo. Estos programas pueden clasificarse en dos grandes grupos: programas más enfocados hacia la inserción laboral, y programas de transición colegio trabajo donde, además del objetivo de formar profesionalmente a los jóvenes para que puedan integrarse en el mercado laboral, existe una intención clara de que estos dispongan también de la oportunidad de reincorporarse al sistema educativo, ya sea obteniendo el graduado de la Educación Secundaria Obligatoria (ESO) o accediendo a la formación profesional de grado medio. Los dos principales programas de este tipo son los Programas de cualificación profesional inicial subvencionados (PCPI S) y el Proyecto de 
formación y de aprendizaje profesional (PCPI FYAP) y son justamente estos los programas que se evalúan en el presente trabajo a través de la lente de los dos objetivos mencionados previamente.

El documento se estructura de la siguiente manera. En la segunda sección se describen los programas. En la tercera se describen las fuentes de información utilizadas y hace una primera exploración de los participantes del programa. La cuarta sección explica la evaluación de impacto. Finalmente, el último capítulo aporta las conclusiones de la evaluación.

\section{Descripción del programa}

Los programas de cualificación profesional inicial (PCPI) están dirigidos a jóvenes de 16 a 25 años que finalizan su recorrido escolar sin haber obtenido el título de graduado en Educación Secundaria Obligatoria (ESO). Los PCPI tienen una duración de un año académico, la oferta se estructura en ámbitos y perfiles profesionales (auxiliar de jardinería, auxiliar de carpintería metálica y PVC, auxiliar en actividades de oficina y en servicios administrativos generales, etc.), y se desarrollan con el doble objetivo de proporcionar a los alumnos un nivel básico de cualificación profesional que les facilite la incorporación al mercado de trabajo y, al mismo tiempo, les ofrezca la opción de recuperar y continuar el itinerario de formación académica, ya sea por vía de la obtención del título de graduado en educación secundaria obligatoria o por la vía de acceso a la formación profesional de grado medio.

Sobre la base de los antiguos programas de garantía social (PGS), los PCPI nacen con la ley orgánica 2/2006, de 3 de mayo, de Educación, la cual encomienda a las administraciones educativas la organización de programas de cualificación profesional inicial destinados al alumnado mayor de 16 años que no haya obtenido el título de graduado de ESO, con los siguientes objetivos: 1) adquisición por parte de los alumnos de competencias profesionales correspondientes a las cualificaciones de nivel 1 del catálogo nacional de cualificaciones profesionales, 2) posibilitar a los alumnos una inserción sociolaboral satisfactoria, y 3) ampliar las competencias básicas de los alumnos para permitirles proseguir estudios en las distintas enseñanzas. Igualmente, en esta ley se estipula que los PCPI deben incluir los tres tipos de módulos siguientes: 
A) Módulos formativos específicos, referidos a las unidades de competencia correspondientes a las cualificaciones de nivel 1 del catálogo nacional de cualificaciones profesionales.

B) Módulos formativos de carácter general, que amplíen las competencias básicas y favorezcan la transición desde el sistema educativo al mundo laboral.

C) Módulos de carácter voluntario, que conduzcan a la obtención del título de graduado en Educación Secundaria Obligatoria (ESO) y que puedan cursarse de forma simultánea, o con posterioridad, a los módulos obligatorios A y B.

En Catalunya, cerca de un cuarta parte del alumnado acaba la ESO sin obtener el graduado correspondiente ${ }^{6}$. La situación de estos jóvenes es problemática, ya que la falta de cualificaciones hace muy difícil que puedan incorporarse al mercado laboral, y al no disponer del graduado de la ESO, tampoco pueden continuar la formación regulada. Para intentar reconducir esta situación, los PCPI ofrecen a estos jóvenes una opción de formación, que se diferencia de la secundaria obligatoria en dos características principales: por una parte, una atención en el proceso formativo más individualizada, con grupos reducidos (con un mínimo de 10 jóvenes y un máximo de 15), un peso elevado de la acción tutorial y orientadora por parte de los docentes y una adaptación flexible a las características y necesidades de cada joven; por otra parte, unos contenidos menos teóricos, estructurados en diferentes especialidades que el joven puede escoger, directamente relacionados con el mundo laboral y con la oportunidad de una primera experiencia laboral mediante prácticas en empresas. La duración de los módulos obligatorios de un PCPI es de un curso escolar y comprende un mínimo de 800 horas y un máximo de 1,100. La formación en centros de trabajo tiene una duración comprendida entre las 150 y las 250 horas. La evaluación del alumno incluye tanto la cualificación final de los módulos formativos como el resultado de la formación en centros de trabajo (apto o no apto). La superación del PCPI requiere que la cualificación final sea igual o superior a 5 y que la valoración de la formación en centros de trabajo sea apto. El alumnado que no supere la evaluación puede continuar la formación y cursar los módulos no superados como máximo durante otro curso académico.

\footnotetext{
${ }^{6}$ La tasa bruta de graduación en ESO era, al final del curso 2007-2008, del 76,3\% en Cataluña y del $71,5 \%$ en el conjunto del Estado. Esta tasa muestra la relación entre el número de graduados en ESO independientemente de su edad, respecto del total de la población en la edad teórica (15 años) de comienzo del último curso de la ESO. (Fuente: Instituto de Evaluación. Sistema estatal de indicadores de la educación, 2011).
} 
Sobre la base de este diseño, se espera que la tasa de retención y graduación de los PCPI sea elevada, y que al finalizar el PCPI los jóvenes: 1) «desarrollen y consoliden la madurez personal [...] mediante la adquisición de actitudes, habilidades y capacidades que les permitan participar como ciudadanos responsables»; 2) adquieran un título y las correspondientes competencias profesionales que les permitan «una inserción social y laboral satisfactoria»; y 3) opten por proseguir su itinerario formativo. En este sentido, los PCPI ofrecen diversas oportunidades. En primer lugar, superar el PCPI supone una oportunidad adicional para acceder a las enseñanzas de formación profesional de grado medio. De forma general, cualquier joven mayor de 17 años que no disponga del graduado en educación secundaria obligatoria tiene la opción de acceder a la formación profesional inicial (CFGM) mediante una prueba de acceso específica. En cualquier caso, los graduados de PCPI parten con toda una serie de ventajas: 1) de forma general, se presupone que han ampliado sus competencias básicas y que esto debería facilitarles la superación de la prueba; 2) están exentos, con carácter general, de la parte científico-técnica de la prueba; 3) se les reconoce la cualificación global del PCPI en el cálculo de la nota final de la prueba; 4) están totalmente exentos de la prueba si la cualificación global del PCPI ha sido igual o superior a ocho. En segundo lugar, los PCPI abren la puerta a obtener el título de graduado en ESO por la vía de cursar y aprobar el módulo $\mathrm{C}$ voluntario. Finalmente, el título de un PCPI da lugar a la consecución del nivel 1 de la estructura del catálogo de cualificaciones profesionales, lo cual constituye un primer capital formativo hacia la obtención de certificados de profesionalidad 2 y $3 .^{7}$

En última instancia, los objetivos estratégicos de los PCPI son la consecución de un mayor nivel formativo y una mejor adaptación, integración y promoción en el mundo laboral de la que se hubiera conseguido en ausencia del programa. Resulta destacable, sin embargo, que los diferentes documentos explicativos y normativas que regulan los PCPI no establecen una prioridad entre estos dos objetivos. En este sentido, el programa no se ubica claramente, al menos sobre la base de sus fundamentos teóricos, ni en el marco de la estrategia work first ni en la de acumulación de capital humano que suelen orientar las políticas activas de empleo.

7 Los certificados de profesionalidad, expedidos por el soc, acreditan las competencias profesionales de la persona, independientemente de dónde se hayan adquirido (experiencia profesional o formación, regulada o no regulada). El reconocimiento de competencia se establece de acuerdo con el catálogo nacional de cualificaciones profesionales (más información en

http://www.oficinadetreball.cat/socweb/opencms/socweb_ca/ciutadans/Certificats_profession alitat.html) 
La implementación y gestión de los PCPI resulta particularmente compleja, en tanto que el programa se estructura en cuatro subprogramas diferentes:

- Los PCPI - Formación y aprendizaje profesional (PCPI-FYAP). En este caso, el Departamento de Educación desarrolla los PCPI en el marco de un convenio con el Servicio de Empleo de Catalunya, que es el que aporta la financiación. Dado que esta financiación procede mayoritariamente del Fondo Social Europeo, se establecen ciertos requisitos, como el que un porcentaje mínimo de alumnos se hayan registrado previamente en el soc como trabajadores en paro.

- Los PCPI públicamente subvencionados (PCPI-S). En este caso, es el sOC el encargado de publicar anualmente una convocatoria de subvenciones en régimen de concurrencia competitiva a la que se pueden presentar centros y entidades de formación inscritos en el Registro de centros y entidades de formación del Servei d'Ocupació de Catalunya y que hayan sido autorizados para impartir un Programa de cualificación profesional inicial por parte del Departamento de Educación (es decir, las entidades deben superar un doble proceso de acreditación para poder optar a las subvenciones). Las entidades beneficiarias pueden ser tanto privadas como administraciones públicas de ámbito local, y los criterios de otorgamiento de las subvenciones son la adecuación de los PCPI presentados a las necesidades del mercado de trabajo en el territorio, la capacidad y calidad de las entidades solicitantes para realizar acciones formativas y la valoración técnica de la metodología propuesta. Igualmente, la orden TRE/37/2010, de 28 de junio, que establece las bases de concesión de subvención de los PCPI, condiciona la cantidad de la subvención al número de alumnos que finalicen la acción formativa, entendido esto último como los casos que hayan asistido al menos a un $75 \%$ del total de la acción formativa. La convocatoria prescribe que los PCPI subvencionados deben ser gratuitos y que el importe de la subvención concedida no puede superar, aisladamente o junto con otras subvenciones, el coste de la actividad formativa. La organización y gestión de cada uno de los cursos subvencionados es autónoma.

- Los PCPI que se desarrollan en el marco del Plan de transición al trabajo (PCPI-PTT). En este caso, corresponde al Departamento de Educación financiar, planificar y gestionar los PCPI conjuntamente con las administraciones locales (ayuntamientos, agrupaciones de ayuntamientos, consejos comarcales y diputaciones). 
- Los PCPI no subvencionados (PCPI-NS). Estos son gestionados e implementados, de forma autónoma, por entidades privadas o administraciones locales, previa autorización por parte del Departamento de Educación, pero sin subvención del SOC. Este es el único caso en que los PCPI no son gratuitos.

Como mencionamos en la introducción, únicamente los dos primeros 'subprogramas' forman parte del presente estudio. De esta forma, la evaluación de impacto se ha realizado sobre los 2,401 participantes que comenzaron el programa PCPI-S durante el curso escolar 2008-2009 y los 1,220 que tomaron parte en alguno de los PCPI-FYAP ofertados durante ese mismo curso.

\section{Fuentes de información utilizadas}

La figura 1 contiene una representación esquemática de las fuentes de datos utilizadas, así como de las preguntas de evaluación que hemos intentado responder a partir de su utilización. Las fuentes de información son aportadas por el Departamento de Empresa y Empleo (DEMO), por el SOC y por el Departamento de Educación de Catalunya. En concreto, las bases de datos utilizadas son las siguientes:

a) Base de datos de integración del soc. Identifica a los participantes de los dos programas para jóvenes que han sido analizados (PCPI y FYAP), a los participantes de otros programas dirigidos en exclusiva a este colectivo, así como a los participantes de otras políticas activas de empleo «generales» en las que también participan jóvenes. Igualmente, identifica el tipo de entidad beneficiaria en que se desarrolla cada uno de los programas (ente local, organismo de la Generalitat o entidad sin ánimo de lucro), la fecha de inicio y finalización de la participación en el programa por parte de los beneficiarios y, finalmente, si los responsables de la actividad valoran positivamente el aprovechamiento por parte de cada uno de los beneficiarios.

b) Base de datos SicAs-SiSPE. Es la base de datos general de los demandantes de empleo de Catalunya. Los registros históricos corresponden al último día laboral de cada mes, desde mayo de 2005. Contiene información sociodemográfica básica (sexo, año de nacimiento, edad, nacionalidad, población de residencia y nivel formativo), sobre las preferencias y disposición a trabajar (restricciones de jornada, disposición a trabajar fuera del municipio o comarca, número de empleos diferentes solicitados y tipo de empleo solicitado), historia de desempleo (fecha de inicio del periodo de 
desempleo actual), historia laboral (declaración del tiempo total trabajado $\mathrm{y}$ declaración del sector del último empleo), así como otros atributos relevantes para el empleo (percepción de la prestación activa, conocimiento de idiomas, declaración de discapacidades, etc.).

\section{c) Base de datos de afiliación a la Seguridad Social y altas de contratos} laborales. Los datos de la seguridad social están estructurados en archivos trimestrales, correspondientes al último día laborable de cada trimestre, contiene información sobre las altas en la Seguridad Social. Al igual que las otras bases de datos, los registros históricos están disponibles desde 2005 y abarcan hasta el primer trimestre de 2013. Los datos de contratos contienen los contratos y prórrogas para cada mes. Aporta también información sobre la entidad contratante, el sector de actividad económica, la localización del lugar de trabajo y el tipo de contrato.

\section{d) Información facilitada por el Departamento de Educación:}

- Relación de personas matriculadas en cursos de formación de personas adultas para obtener el Graduado en Educación Secundaria Obligatoria (GESO).

- Relación de personas matriculadas en Ciclos Formativos de Grado Medio (cfgm)

- Relación de personas que han solicitado el título de geso

- Relación de personas que han obtenido el título de técnico - CFGM 
Figura 1: fuentes de información y preguntas de evaluación

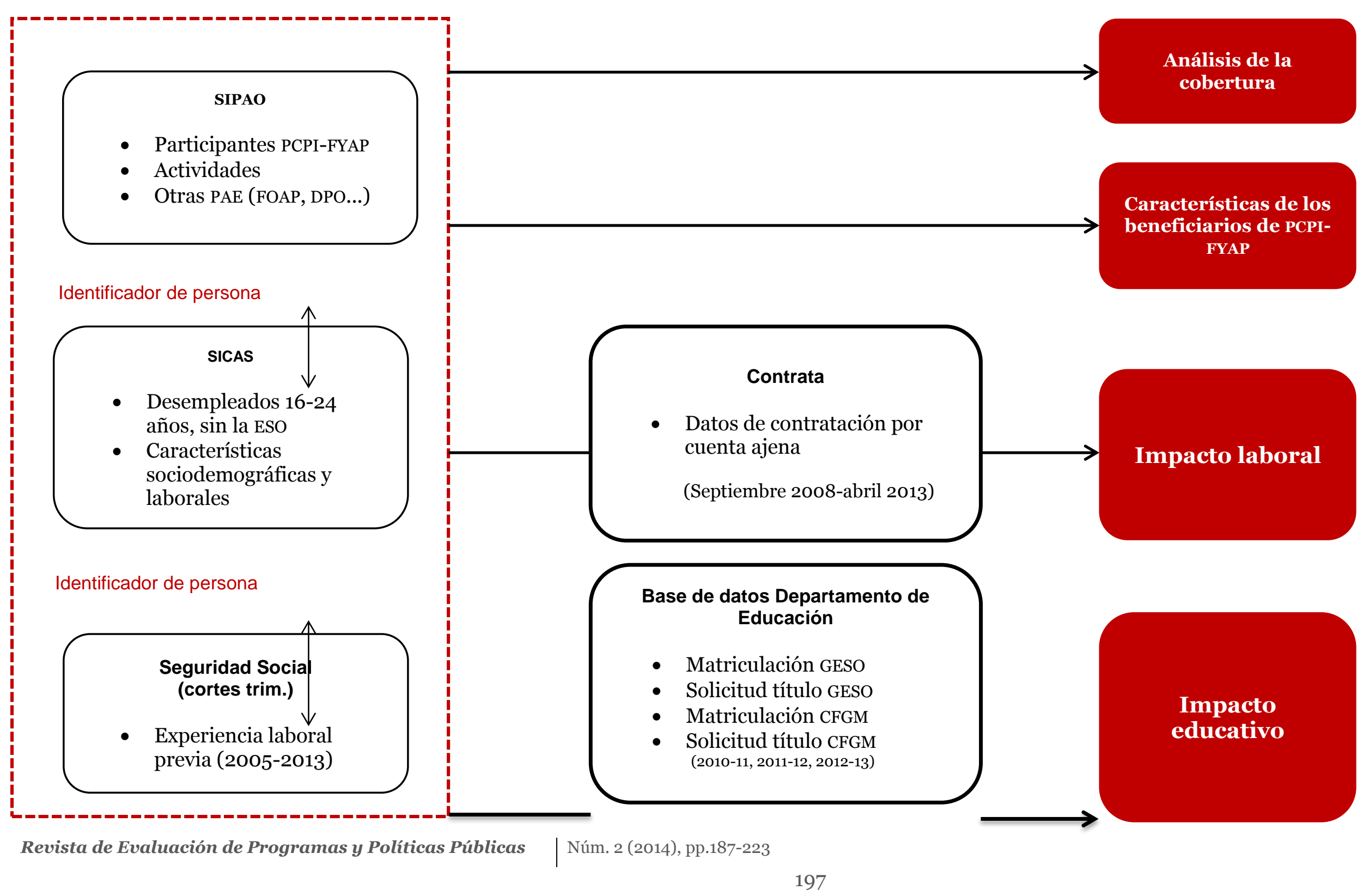




\section{Selección y acceso de los participantes}

Una cuestión crucial a la hora de evaluar cualquier programa que tenga por objetivo proporcionar un determinado servicio a una población diana - como es el caso de los PCPI-S y PCPI-FYAP-, consiste en establecer qué factores determinan en la práctica que unas personas accedan a ellos y otras no. Incluso cuando existen criterios de acceso regulados normativamente, resulta fundamental contrastar empíricamente si estos criterios se cumplen, así como detectar otros factores que puedan estar influyendo sobre el proceso de acceso al programa. En el caso que nos ocupa, las posibles discrepancias entre los criterios «teóricos» de acceso al programa y la manera en que estos acaban operando en la práctica pueden tener que ver con: primero, la participación en programas no obligatorios no depende solamente de la voluntad de las administraciones públicas de proporcionárselos a una determinada población diana, sino también de la disposición de los potenciales beneficiarios a participar en ellos; segundo, aunque un programa tenga explicitados criterios de acceso, son los responsables y los trabajadores que lo implementan los que acaban modulando la demanda en la práctica y, por tanto, pueden valorar otros factores además de los que estipula la normativa.

En el caso de los PCPI, la normativa establece que la población diana del programa está constituida por mayores de 16 años hasta un máximo de 21 años, en el caso de los PCPI-FYAP, y de 25 en el de los PCPI-S, que no hayan obtenido el título de la ESO y que estén inscritos en la Oficina de Empleo como demandantes de trabajo ${ }^{8}$. No define, en cambio, los criterios para priorizar candidatos en caso de que exista un exceso de demanda. ¿A qué tipo de joven están llegando en la práctica los PCPI? ¿Qué características personales influyen en la participación, bien porque predisponen a los jóvenes a querer participar en un PCPI o bien porque los responsables del programa las tienen en cuenta a la hora de seleccionar candidatos?

\footnotetext{
${ }^{8}$ Esto último pueden hacerlo después de tomar la decisión de participar en el programa
} 


\subsection{Similitudes $y$ diferencias entre participantes $y$ no participantes en los PCPI}

La tabla 1 compara las características de los participantes en PCPI-S y PCPI-FYAP, en cada, con las de los parados registrados en el SOC, sin el título de la ESO y en el rango de edad correspondiente - esto es, elegibles en términos del programa - pero que no participaron. Las primeras cuatro columnas muestran la distribución de frecuencias de las variables sociodemográficas para los cuatro grupos de jóvenes siguientes: 1) participantes en los PCPI-S; 2) no participantes en PCPI-S; 3) participantes en los PCPI-FYAP; y 4) no participantes en PCPI-FYAP.

De acuerdo al análisis, los participantes en PCPI-S (respecto al resto de jóvenes desempleados del mismo rango de edad y sin la ESO que no participan) presentan una menor proporción de hombres y de inmigrantes extracomunitarios, una mayor proporción de personas de entre 16 y 19 años. Al mismo tiempo presentan una mayor frecuencia de jóvenes con baja empleabilidad, sin ningún tipo de experiencia laboral previa y, en caso de tenerla, con un menor número de trimestres trabajados. Los participantes son tienen una proporción mayor de desempleados de corta duración (menos de 3 meses), un menor dominio del catalán y del inglés y una menor flexibilidad a la hora de aceptar un trabajo (es superior el porcentaje de aquellos que solicitan un solo empleo, y también de los que solamente aceptarían trabajar en su misma provincia). Finalmente, vienen proporcionalmente más de municipios grandes (más de 150.000 habitantes), de la provincia de Barcelona y, especialmente, del Baix Llobregat y de Barcelona ciudad.

En el caso de los PCPI-FYAP, el perfil de los participantes es bastante similar al de los participantes de los PCPI-S, con algunas excepciones. Respecto a aquellos jóvenes no participantes que cumplen los requisitos para participar en el programa, los inscritos en los PCPI-FYAP presentan un porcentaje muy superior de hombres, una frecuencia de inmigrantes extracomunitarios más elevada y un porcentaje de la demarcación de Tarragona más elevado. 
Tabla 1: comparación de las características entre participantes y no participantes

\begin{tabular}{|c|c|c|c|c|}
\hline & \multicolumn{2}{|c|}{ PCPI-S } & \multicolumn{2}{|c|}{ PCPI-FYAP } \\
\hline & $\begin{array}{c}\text { No } \\
\text { participantes }\end{array}$ & Participantes & $\begin{array}{c}\text { No } \\
\text { participantes }\end{array}$ & Participantes \\
\hline \multicolumn{5}{|l|}{ Sexo } \\
\hline Hombre & $63,8 \%$ & $60,5 \%$ & $64,6 \%$ & $93,7 \%$ \\
\hline \multicolumn{5}{|l|}{ Empleabilidad $^{(1)}$} \\
\hline Subsidio RAI & $0,4 \%$ & $0,1 \%$ & $0,3 \%$ & $0,0 \%$ \\
\hline Muy baja & $2,1 \%$ & $0,9 \%$ & $1,7 \%$ & $0,8 \%$ \\
\hline Baja & $41,4 \%$ & $68,6 \%$ & $44,3 \%$ & $65,8 \%$ \\
\hline Media & $41,7 \%$ & $26,0 \%$ & $41,1 \%$ & $28,7 \%$ \\
\hline Alta & $14,4 \%$ & $4,4 \%$ & $12,7 \%$ & $4,8 \%$ \\
\hline Discapacidad & $1,1 \%$ & $1,6 \%$ & $1,2 \%$ & $0,6 \%$ \\
\hline \multicolumn{5}{|l|}{ Nacionalidad } \\
\hline Español & $62,1 \%$ & $72,4 \%$ & $67,1 \%$ & $58,1 \%$ \\
\hline Comunitario & $5,8 \%$ & $2,1 \%$ & $3,7 \%$ & $1,8 \%$ \\
\hline Extracomunitario & $32,1 \%$ & $25,5 \%$ & $29,2 \%$ & $40,2 \%$ \\
\hline \multicolumn{5}{|l|}{ Edad } \\
\hline $16-18$ & $21,6 \%$ & $88,8 \%$ & $38,2 \%$ & $89,9 \%$ \\
\hline 19-21 & $34,7 \%$ & $9,3 \%$ & $61,8 \%$ & $10,1 \%$ \\
\hline 22-24 & $43,7 \%$ & $1,9 \%$ & & \\
\hline \multicolumn{5}{|l|}{$\begin{array}{l}\text { Antigüedad en el } \\
\text { paro }\end{array}$} \\
\hline o - 3 meses & $62,0 \%$ & $79,8 \%$ & $65,9 \%$ & $87,4 \%$ \\
\hline 4-12 meses & $31,6 \%$ & $18,5 \%$ & $29,3 \%$ & $11,3 \%$ \\
\hline$>12$ meses & $6,4 \%$ & $1,7 \%$ & $4,8 \%$ & $1,3 \%$ \\
\hline
\end{tabular}


Comparación de las características entre participantes y no participantes (cont.)

\begin{tabular}{|c|c|c|c|c|}
\hline & \multicolumn{2}{|c|}{ PCPI-S } & \multicolumn{2}{|c|}{ PCPI-FYAP } \\
\hline & $\begin{array}{c}\text { No } \\
\text { participantes }\end{array}$ & Participantes & $\begin{array}{c}\text { No } \\
\text { participantes }\end{array}$ & Participantes \\
\hline \multicolumn{5}{|l|}{ Empleos solicitados } \\
\hline 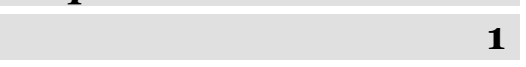 & $34,6 \%$ & $76,6 \%$ & $39,9 \%$ & $78,3 \%$ \\
\hline 2 & $19,3 \%$ & $8,7 \%$ & $18,0 \%$ & $8,1 \%$ \\
\hline 3 & $15,6 \%$ & $6,1 \%$ & $14,6 \%$ & $5,0 \%$ \\
\hline 4 & $12,1 \%$ & $3,4 \%$ & $10,9 \%$ & $3,2 \%$ \\
\hline 5 & $9,1 \%$ & $2,7 \%$ & $8,4 \%$ & $3,0 \%$ \\
\hline 6 & $9,3 \%$ & $2,6 \%$ & $8,2 \%$ & $2,4 \%$ \\
\hline \multicolumn{5}{|l|}{ Población } \\
\hline$<=1000$ & $1,2 \%$ & $1,1 \%$ & $1,2 \%$ & $1,3 \%$ \\
\hline De 1001 a 5000 & $6,3 \%$ & $4,9 \%$ & $6,4 \%$ & $7,3 \%$ \\
\hline De 5001 a 20000 & $17,7 \%$ & $16,8 \%$ & $17,1 \%$ & $14,9 \%$ \\
\hline De 20001 a 75000 & $29,7 \%$ & $23,9 \%$ & $29,3 \%$ & $26,1 \%$ \\
\hline De 75001 a 150000 & $18,4 \%$ & $17,2 \%$ & $18,1 \%$ & $19,1 \%$ \\
\hline$<150000$ & $26,7 \%$ & $36,1 \%$ & $27,9 \%$ & $31,4 \%$ \\
\hline \multicolumn{5}{|l|}{ Jornada } \\
\hline Jornada parcial & $0,9 \%$ & $2,2 \%$ & $1,2 \%$ & $1,2 \%$ \\
\hline Jornada completa & $1,2 \%$ & $1,7 \%$ & $1,5 \%$ & $2,0 \%$ \\
\hline Indiferente & $97,9 \%$ & $96,1 \%$ & $97,2 \%$ & $96,7 \%$ \\
\hline \multicolumn{5}{|l|}{ Lengua } \\
\hline Catalán & $13,5 \%$ & $11,0 \%$ & $14,0 \%$ & $7,7 \%$ \\
\hline Inglés & $67,0 \%$ & $58,4 \%$ & $68,6 \%$ & $55,2 \%$ \\
\hline \multicolumn{5}{|l|}{ Trabajo } \\
\hline Experiencia previa & $70,1 \%$ & $10,4 \%$ & $56,4 \%$ & $10,5 \%$ \\
\hline $\begin{array}{r}\text { Num trim. trabajados } \\
\text { previamente }\end{array}$ & 6,58 & 0,41 & 4,20 & 0,34 \\
\hline
\end{tabular}




\begin{tabular}{|c|c|c|c|c|}
\hline & \multicolumn{2}{|c|}{ PCPI-S } & \multicolumn{2}{|c|}{ PCPI-FYAP } \\
\hline & $\begin{array}{c}\text { No } \\
\text { participantes }\end{array}$ & Participantes & $\begin{array}{c}\text { No } \\
\text { participantes }\end{array}$ & Participantes \\
\hline \multicolumn{5}{|l|}{ Provincia } \\
\hline Barcelona & $66,5 \%$ & $81,3 \%$ & $67,4 \%$ & $69,2 \%$ \\
\hline Gerona & $13,6 \%$ & $3,8 \%$ & $13,2 \%$ & $8,1 \%$ \\
\hline Lérida & $6,4 \%$ & $2,4 \%$ & $5,8 \%$ & $5,2 \%$ \\
\hline Tarragona & $13,6 \%$ & $12,6 \%$ & $13,5 \%$ & $17,5 \%$ \\
\hline \multicolumn{5}{|l|}{ Servicio territorial } \\
\hline Baix Llobregat & $19,2 \%$ & $22,0 \%$ & $19,4 \%$ & $17,5 \%$ \\
\hline Barcelona Ciudad & $11,7 \%$ & $17,2 \%$ & $11,9 \%$ & $18,3 \%$ \\
\hline Gerona & $12,2 \%$ & $3,4 \%$ & $11,7 \%$ & $6,6 \%$ \\
\hline Lérida & $6,4 \%$ & $2,4 \%$ & $5,8 \%$ & $5,2 \%$ \\
\hline Tarragona & $11,1 \%$ & $10,9 \%$ & $11,0 \%$ & $12,8 \%$ \\
\hline Terres de l'Ebre & $2,5 \%$ & $1,6 \%$ & $2,5 \%$ & $4,7 \%$ \\
\hline Vallès Occidental & $16,1 \%$ & $18,5 \%$ & $16,7 \%$ & $17,1 \%$ \\
\hline Vallès Oriental & $19,5 \%$ & $23,6 \%$ & $19,4 \%$ & $16,3 \%$ \\
\hline \multicolumn{5}{|l|}{ Ámbito demanda } \\
\hline $\begin{array}{r}\text { Dentro de la } \\
\text { provincia }\end{array}$ & $88,1 \%$ & $92,7 \%$ & $88,4 \%$ & $88,8 \%$ \\
\hline
\end{tabular}

(1) El nivel de empleabilidad es una variable sintética que comprende tres medidas diferentes: antigüedad de la demanda de empleo, ámbito territorial de la búsqueda de empleo y número de empleos solicitados (para obtener más detalles, véase Toharía et al., 2006). 


\subsection{Los determinantes de la participación en los PCPI}

Los resultados de la tabla anterior proporcionan una información valiosa sobre las características de los beneficiarios de unos programas y otros, pero están sometidos a una limitación importante: no permiten descartar que las asociaciones detectadas sean espurias, es decir, que haya una tercera variable que explique la relación que se observa entre el hecho de participar en los programas y una determinada característica personal: en el caso del pcpi-fyap, por ejemplo, la asociación detectada entre ser inmigrante y participar en el programa puede no ser debida a la condición de ser inmigrante en sí misma, sino al hecho de que la mayoría de participantes son hombres y, por otra parte, la mayoría de inmigrantes también lo son. Por este motivo se ha estimado un modelo logit que permite calcular de manera simultánea la asociación neta (no mediada por terceras variables) entre cada una de las variables analizadas y el hecho de participar o no en estos dos programas.

Los resultados de los diversos logits estimados se muestran en los gráficos 1 y 2 , correspondientes al año 2008. Los valores que aparecen representan el efecto medio que tiene modificar el valor de cada una de las variables sobre la probabilidad de que una persona sea participante. Por ejemplo, si nos fijamos en los determinantes de la participación en el pcpi-s (gráfico 1), un valor de -4,5 de la variable «más de 12 meses en paro» significa que, de media, un joven desempleado de larga duración tiene una probabilidad 4,5 puntos porcentuales más pequeña de participar en un pcpi que otra persona, igual en el resto de características, pero que «lleva menos de tres meses en paro» (categoría de referencia).9 El color rojo de las barras muestra que el efecto medio es estadísticamente significativo, en oposición al color blanco, que representa efectos medios no significativos.

Los principales resultados a destacar en lo que respecta a la participación en los PCPI-S son los siguientes:

- La probabilidad de participar en este programa disminuye con la edad de los individuos, con la existencia de una experiencia laboral previa y también con el

\footnotetext{
${ }^{9} \mathrm{El}$ efecto medio de cada variable debe entenderse siempre respecto a la categoría de referencia, que es la que no aparece en el gráfico (por ejemplo, el efecto medio de ser extracomunitario debe entenderse respecto del hecho de ser español, a igualdad del resto de características).
} 
hecho de ser hombre o inmigrante, aunque la magnitud de estos dos últimos efectos es notablemente inferior a la de los dos primeros.

- La probabilidad de participar aumenta 4,4 puntos porcentuales en los jóvenes que demandan un solo tipo de empleo.

- En lo que respecta al efecto de las variables de carácter territorial, todos los jóvenes de fuera de la ciudad de Barcelona tienen, a igualdad en el resto de características, menos probabilidades de participar en un PCPI, especialmente en los municipios correspondientes a los Servicios Territoriales de Gerona y Lérida. Igualmente, a igualdad en el resto de características, los jóvenes que viven en municipios de más de 150.000 habitantes tienen probabilidades más bajas de participar en este tipo de programas que los jóvenes de ciudades menos pobladas e, incluso, de pueblos pequeños (menos de 1.000 habitantes).

En relación con los determinantes de la participación en los PCPI-FYAP (gráfico 1), destacan los resultados siguientes:

- La edad y la experiencia laboral hacen disminuir la probabilidad de participación, igual que en el caso de los PCPI-S, pero, por el contrario, el hecho de ser hombre aumenta la probabilidad de cursar un PCPI-FYAP. El hecho de ser inmigrante no tiene ningún efecto. 
Gráfico 1: determinantes de la participación en los PCPI-S, curso 2008-2009

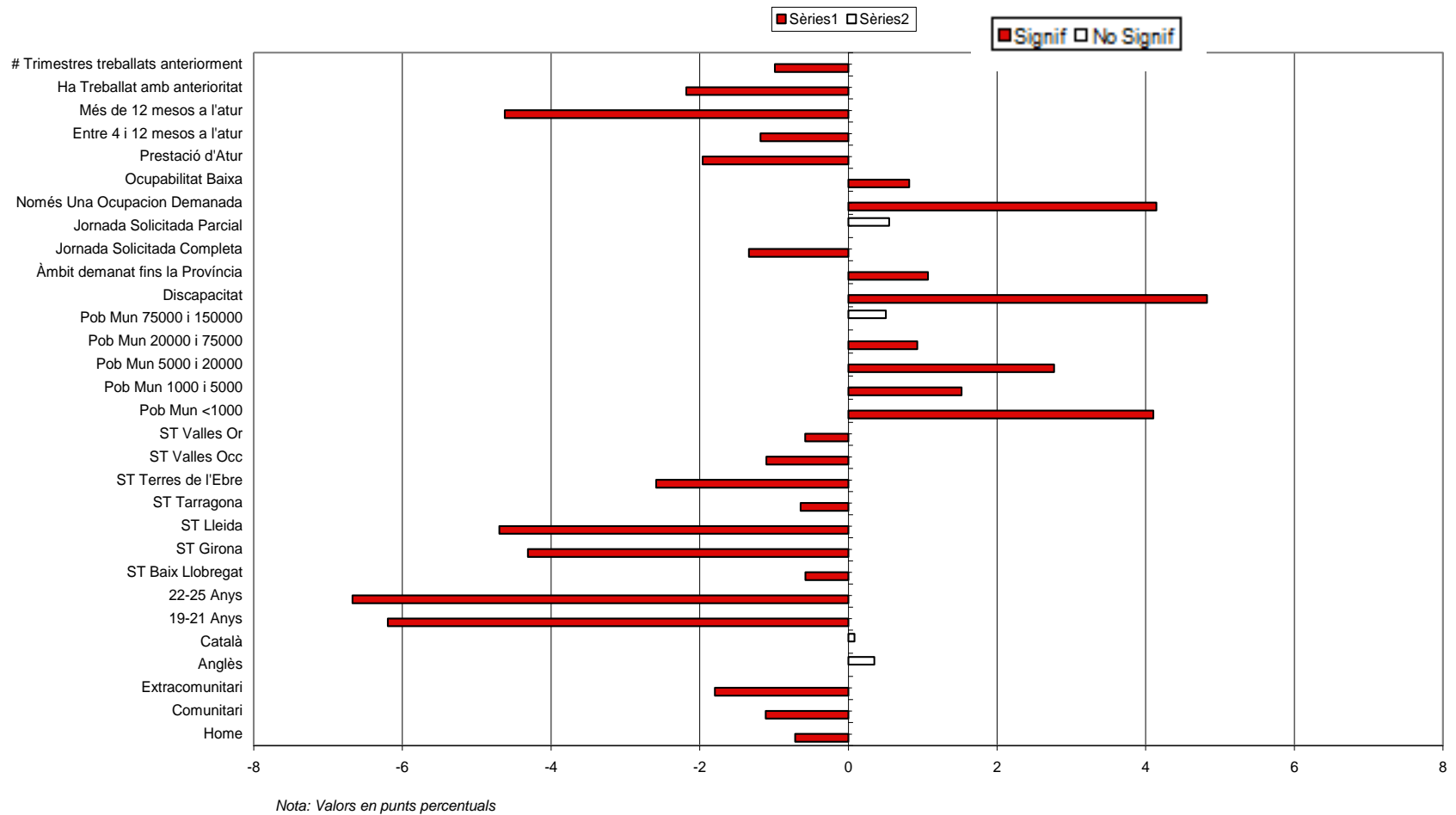

Gráfico 2: determinantes de la participación en los PCPI-FYAP, curso 2008-2009

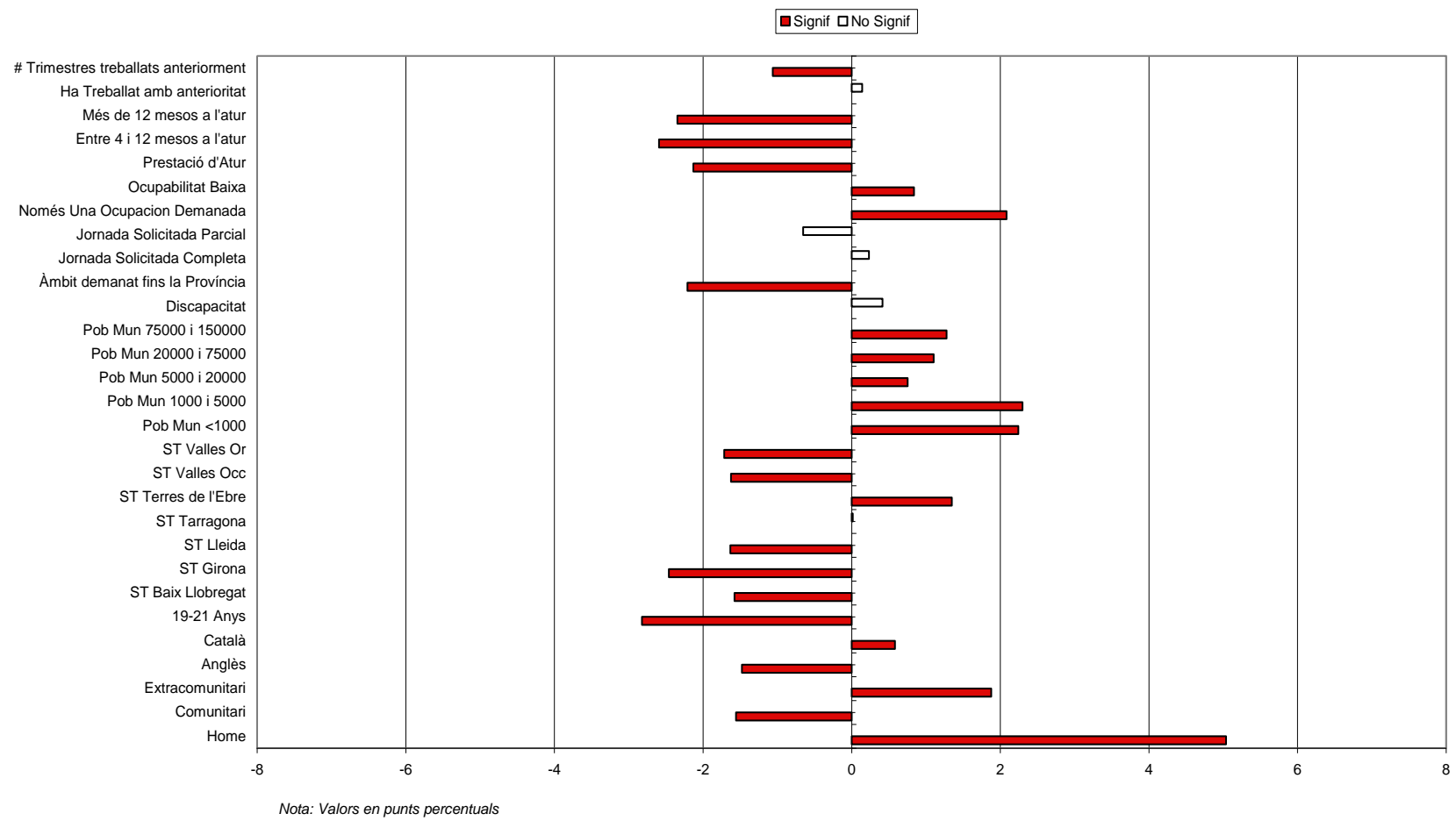




\section{Evaluación del impacto de los PCPI}

El propósito de esta sección capítulo es desentrañar los posibles impactos educativos y laborales de los PCPI-S y PCPI-FYAP. En concreto, las preguntas de evaluación que aquí se plantean son:

- ¿Cuál es el impacto de estos programas sobre la probabilidad de que los jóvenes que participan en ellos vuelvan después al sistema educativo?

- ¿La participación en estos programas aumenta la probabilidad de que los jóvenes desempleados encuentren posteriormente un trabajo?

- ¿ंTienen estos programas un impacto diferencial, tanto en términos de inserción laboral como de retorno al sistema educativo, según el tipo de beneficiario (chicos o chicas, grupos de edad, etc.)?

Sin embargo, antes de entrar a explicar los detalles del análisis realizado, conviene entender qué implica medir el impacto de un programa. El reto consiste en averiguar en qué medida se han producido cambios entre los participantes en los resultados de interés, tales como la inserción laboral o el retorno al sistema educativo, que sean atribuibles causalmente al programa. En otras palabras, se trata de comparar la inserción laboral de los participantes en el programa con la que ellos mismos habrían logrado en caso de no haber participado (el denominado contra-factual). No obstante, dado que unos mismos jóvenes no pueden participar y no participar en el programa simultáneamente, esta relación causal se infiere en la práctica comparando los resultados de un conjunto de participantes (grupo de tratamiento) y otro de no participantes con características muy similares (grupo de comparación o de control). En el caso que nos ocupa, como es habitual en la evaluación de políticas activas de empleo, hemos basado nuestras estimaciones de impacto en la aplicación de la técnica conocida como matching. En el apartado siguiente se explican de forma sucinta sus características. $^{10}$

\footnotetext{
${ }^{10}$ Los lectores interesados en profundizar en estas cuestiones pueden consultar la guía Evaluar el impacto de las políticas activas de empleo (Casado y Todeschini, 2013), publicada recientemente por Ivàlua en el marco de otro encargo del soc.
} 


\subsection{La medida del impacto: matching}

La técnica del matching trata de estimar el impacto de un programa sobre sus participantes mediante la definición, a posteriori y con ayuda de la estadística, de un grupo de comparación de no participantes que se parezca lo más posible a los beneficiarios del programa que se intenta evaluar. Para ello, el método busca para cada uno de los participantes una pareja, o match, que sea lo más parecida posible en todas aquellas variables (personales, familiares y de entorno) que pueden influir tanto en la decisión de participar en el programa como en los resultados de interés.

En nuestro caso, a la hora de emparejar a los participantes de ambos programas con jóvenes desempleados no participantes, las características que hemos podido tener en cuenta para garantizar la similitud entre unos y otros son las contenidas en la base de datos del SICAS, ${ }^{11}$ que incluyen factores sociodemográficos básicos (sexo, año de nacimiento, edad, nacionalidad, población de residencia y nivel formativo), preferencias y disposición a trabajar (restricciones de jornada, disposición a trabajar fuera del municipio o comarca, número de empleos distintos solicitados y tipo de empleo solicitado), historia de paro (fecha de inicio del periodo de desempleo actual), así como otros atributos relevantes para el empleo (percepción de la prestación de desempleo, conocimiento de idiomas, declaración de discapacidades, etc.). También hemos podido disponer de información sobre la experiencia laboral previa al inicio del programa, medida a partir de los cortes trimestrales de la Seguridad Social y las contrataciones mensuales de Contrata ${ }^{12}$.

\subsection{Calidad del matching}

Lamentablemente las bases de datos utilizadas no contienen información relativo a variables que pueden ser relevantes para nuestro análisis, como por ejemplo la estructura del hogar (hijos menores, edad del hijo más pequeño, monoparentalidad,

\footnotetext{
${ }^{11}$ Las variables que se utilizaron para el emparejamiento mediante la técnica del matching son las siguientes:

${ }^{12}$ Por una cuestión de espacio, no hemos incluido en este documento las tablas con el algoritmo utilizado, las variables utilizadas en cada caso, la disminución de la diferencia entre tratados y no tratados en los observables a partir del matching o el resultado del test de Becker y Caliendo (2007) sobre sensibilidad de los resultados del matching a sesgo de no observables. No obstante, dicha información puede encontrarse en el informe completo publicado en la página web de Ivàlua, www.ivalua.cat
} 
etc.), nivel educativo de los padres, la historia completa de desempleo (número y duración de los periodos anteriores de desempleo), historia laboral anterior previa a 2005 o el tiempo que resta hasta agotar la prestación o el subsidio de desempleo. No obstante, un dato interesante es que las personas que utilizamos como controles son aquellas personas que cumplían los requisitos para participar al momento del programa. Ello quiere decir que se habían inscrito como demandantes de empleo en la oficina de desempleo, lo cual indica una motivación a conseguir trabajo, similar a la que pueden tener los que acaban participando.

\section{5•3. Resultados analizados y desagregación de los impactos}

Los resultados sobre los que hemos calculado la existencia de diferencias después del tratamiento entre participantes y no participantes son los que se muestran en la tabla 1, y comprenden resultados tanto laborales como educativos. En concreto, en lo que respecta a los primeros, hemos construido a partir de los datos de contratación mensual (Contrata) tres indicadores: 1) la participación laboral mensual, que mide simplemente si la persona ha trabajado o no durante cada uno de los meses comprendidos entre el momento en que los dos programas finalizaron (octubre de 2009) y el mes de abril de 2013; 2) el número acumulado de meses trabajados, que se construye sumando los valores del anterior indicador a lo largo de la ventana temporal mencionada; y 3) el número máximo de meses trabajados consecutivamente, que de nuevo se construye a partir del primer indicador. A su vez, en lo que respecta a los resultados educativos, hemos medido a partir de las bases de datos del Departamento de Educación los tres indicadores siguientes para los diversos cursos comprendidos entre el curso 2009-10 y el 2012-13: 1) estar matriculado en un centro educativo para obtener el ges, 2) estar matriculado para completar un CFGM, 3) haberse sacado efectivamente el graduado en ESO, y 4) haberse graduado de un CFGM; en estos dos últimos casos, el último curso sobre el que se dispone de información es el 2011-12, ya que en el momento de escribir estas líneas, el Departamento de Educación no disponía todavía de los datos de graduación correspondientes al curso 2012-2013.

En particular, hemos analizado POSIBLES efectos diferenciados según el sexo de los participantes y su edad. Hay que decir que, en el caso de los PCPI-FYAP, la desagregación del análisis es menos exhaustivo por las propias características del programa: por una parte, el límite de edad se situaba en los 21 años y, por otra, el porcentaje de chicas que 
estaban matriculadas es tan bajo que, desafortunadamente, no resulta posible desagregar sus impactos.

Finalmente, conviene aclarar que la estimación de los impactos se ha realizado considerando dos modalidades de participación en los programas. La primera abarca a todos aquellos jóvenes que inician el programa, mientras que la segunda considera como participantes solamente a quienes lo concluyen. En el primer caso, el impacto que se estima se denomina en inglés INTENTION to treat, ya que los participantes sobre los que se evalúa la efectividad del programa incluye a todos los que comenzaron, al margen de que algunos lo abandonen más tarde. En cambio, cuando se utiliza la segunda definición de participación, el estimador de impacto se denomina on the treated, ya que lo que mide es el efecto del programa sobre las personas que lo completan. Es importante destacar que ambos tipos de impacto aportan información relevante sobre el programa. En concreto, no basta con fijarse únicamente en el impacto sobre los que acaban el programa - como suele ser habitual-, por la sencilla razón de que el objetivo que perseguía la intervención era mejorar a todos los que lo empezaron.

\subsection{Los impactos de los PCPI-S}

\subsubsection{Impacto laboral}

Los gráficos 3 i 4 tienen el resultado de los dos análisis laborales principales ${ }^{13}$. En particular, los gráficos muestran el punto medio y el intervalo de confianza del efecto que supone participar en el programa respecto a no hacerlo, medido en puntos porcentuales de diferencia (por ejemplo, un 10,1 significa que los participantes tienen una participación laboral 10,1 puntos por encima de los no participantes). El efecto resulta estadísticamente significativo cuando tanto el punto máximo como el mínimo del intervalo de confianza están por encima (impacto positivo) o por debajo (impacto negativo) del eje de ordenadas, mientras que si ninguno de ellos cruza el eje, el impacto del programa se considera nulo.

\footnotetext{
${ }^{13}$ En el Anexo B del informe completo publicado en la web de Ivàlua (http://ves.cat/isbV) están la totalidad de los resultados contenidos, así como un índice interactivo que permite una rápida localización de cada uno de ellos.
} 
Así pues, según los análisis realizados, se constata lo siguiente en lo que respecta a los impactos laborales de los PCPI-S:

- El programa tiene impactos negativos sobre la participación laboral mensual de los que lo inician, fundamentalmente durante la duración del programa y los primeros meses después de la finalización del programa (lock-in effect), que se convierten en impactos nulos a medida que el tiempo avanza (gráfico 3). De hecho, si consideramos la totalidad del periodo analizado, los impactos acumulados son siempre negativos (ya sea medido en total de meses acumulados trabajando y número máximo de meses trabajados en forma consecutiva).

- En cambio, en lo que respecta a las mujeres y a los participantes más jóvenes (16-18 años), se registran impactos positivos en lo que respecta a las tasas mensuales de participación laboral durante el último año de observación (gráficos 4 y 5), aunque en ningún caso consiguen revertir el lock-in effect de los primeros meses, ya que los impactos en términos acumulados son siempre nulos cuando se considera la totalidad del periodo de análisis.

Finalmente, en lo que respecta a los impactos para aquellos jóvenes que completan la totalidad del programa, los efectos sobre la participación laboral mensual son positivos durante los últimos meses del periodo de análisis (gráfico 2), pero, de nuevo, no son lo bastante importantes como para revertir el lock-in effect cuando se considera la totalidad del periodo de análisis. 
Gráfico 3: participación laboral mensual (\%), impacto participantes iniciales

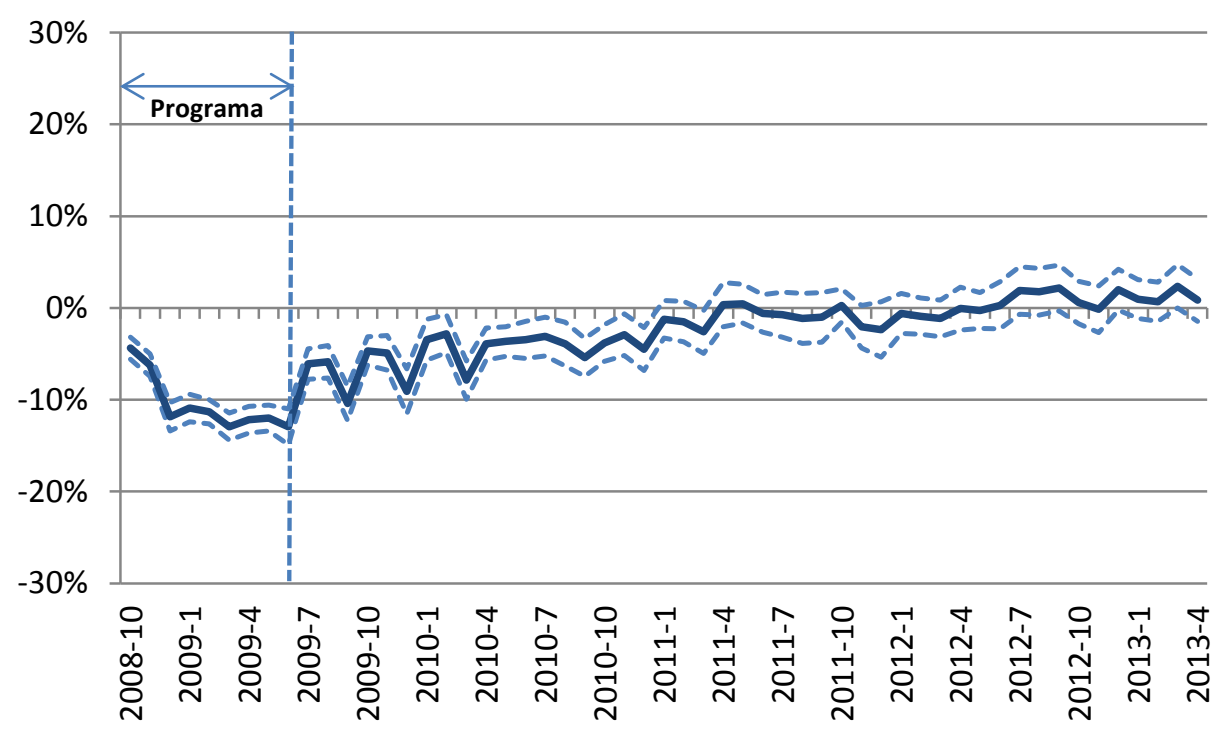

Gráfico 4: participación laboral mensual (\%), impacto participantes que acaban

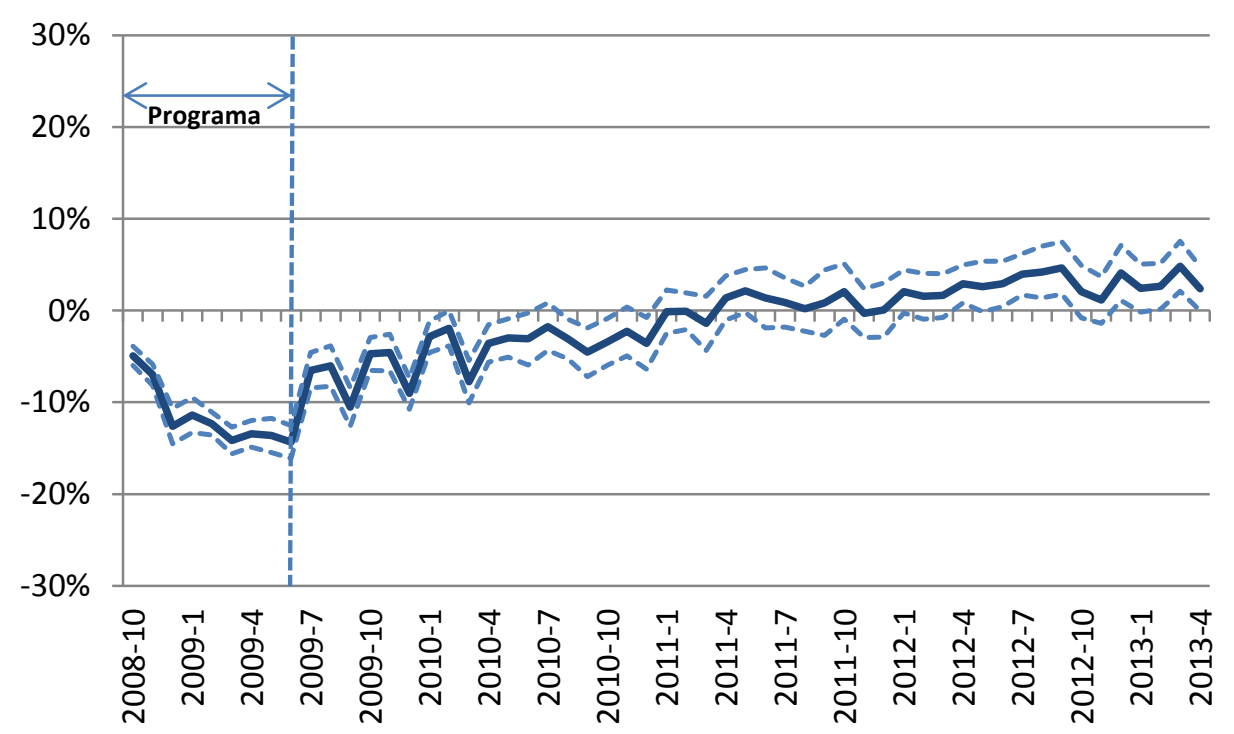




\subsubsection{Impacto educativo}

Los gráficos 5 y 6 contienen la magnitud de los impactos educativos estimados para el conjunto de los participantes iniciales y para aquellos que terminan el programa, respectivamente. A la vista de estos resultados puede afirmarse que:

- El programa tiene efectos positivos sobre la vuelta a los estudios para la consecución del título de la ESO, tanto en términos de matriculación como de graduación efectiva, aunque la magnitud de los impactos es muy modesta (2-3 puntos porcentuales) en ambos casos.

- También se observan impactos positivos muy importantes, de hasta 20 puntos porcentuales, respecto del grupo de comparación, en lo que respecta a la matriculación en los CFGM. No obstante, esta mayor cantidad de matriculaciones no se traduce en una tasa de graduaciones más elevada, ya que no se detectan efectos positivos en ninguno de los casos analizados.

- La totalidad de los impactos educativos mencionados tiene su origen en los jóvenes de entre 16 y 18 años, ya que para los jóvenes de entre 19 y 21 años la mayoría de efectos son nulos (excepto la matriculación en CFGM), e incluso llegan a ser negativos en el caso de los jóvenes de 22 años o más ${ }^{14}$.

Finalmente, con respecto al conjunto de participantes que inician el programa, el hecho de haberlo acabado no tiene ningún impacto diferencial sobre los resultados educativos. Igualmente, en lo que respecta al análisis desagregado de chicos y chicas, los impactos tampoco presentan diferencias destacables.

\footnotetext{
${ }^{14}$ Este gráfico puede encontrarse también en el informe publicado en la web de Ivàlua.
} 
Gráfico 5: matriculación y graduación ESO y CFGM (\% acumulado*), participantes iniciales

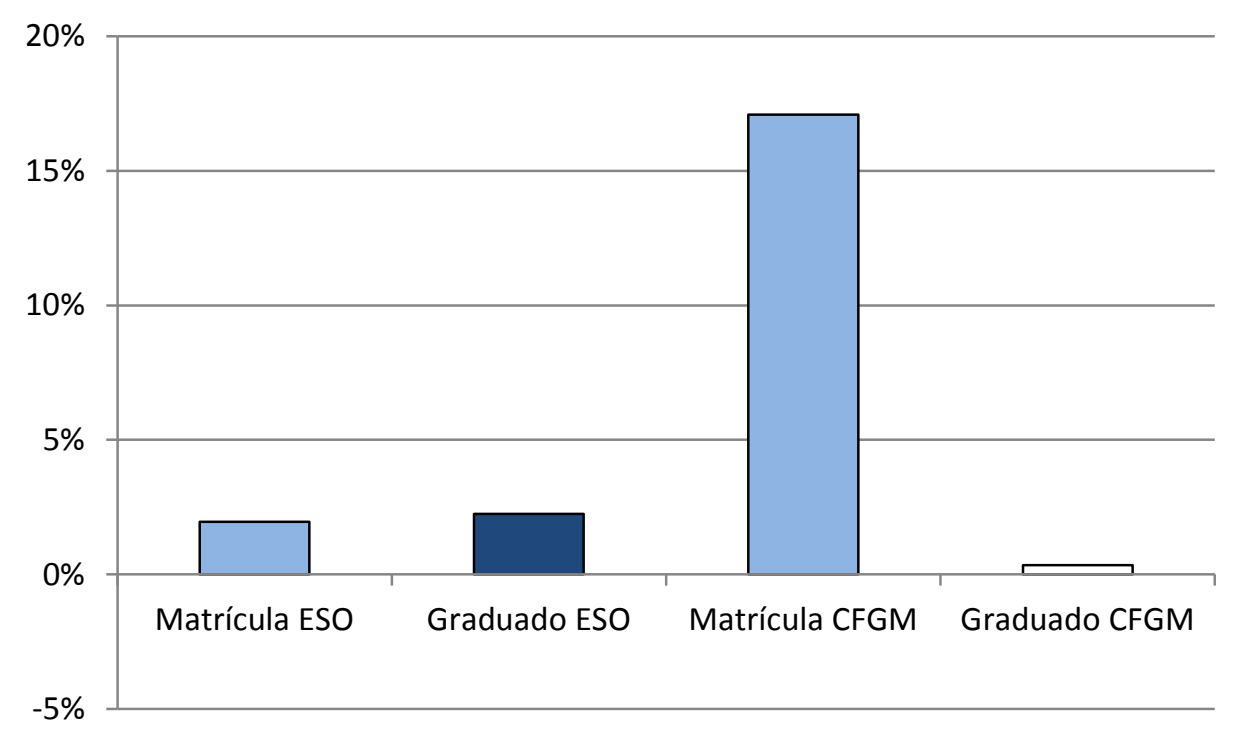

* Cursos 2009-2010 a 2012-2013 (2011-2012, en lo que respecta a la graduación)

Gráfico 6: matriculación y graduación ESO y CFGM (\% acumulado*), participantes que acaban

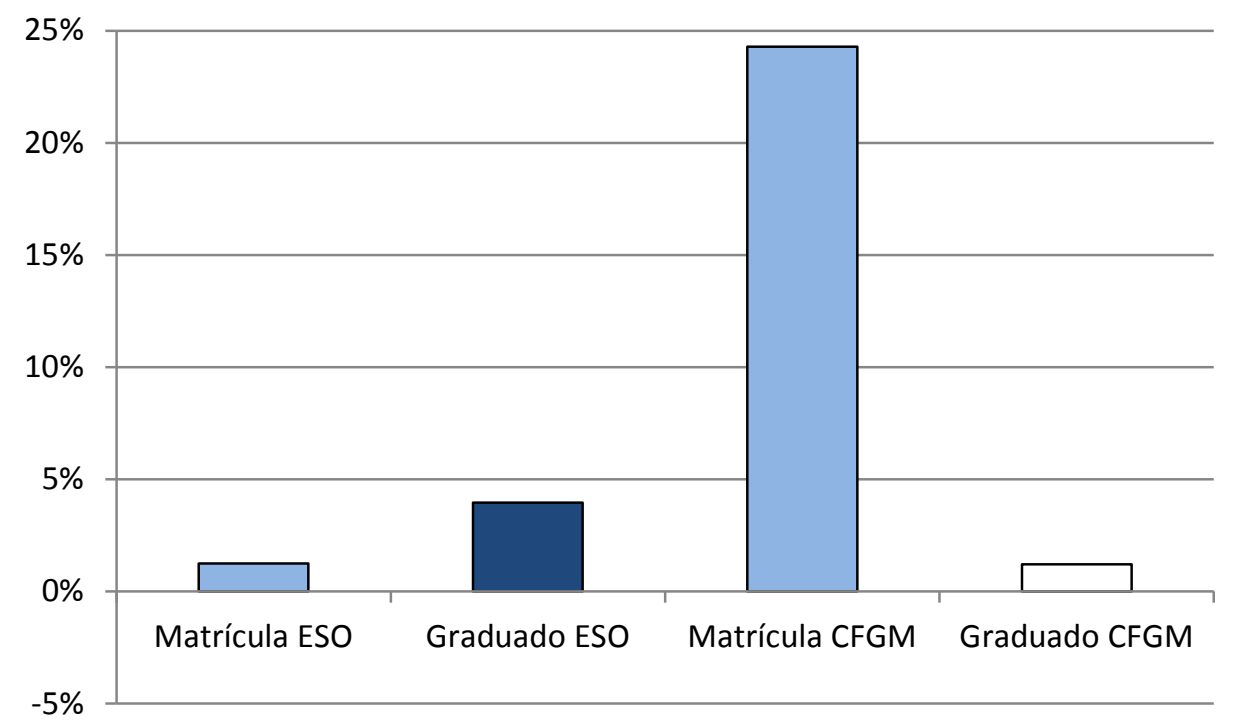




\subsection{Los impactos de los PCPI-FYAP}

\subsubsection{Impacto laboral}

Como en el caso anterior, los gráficos 7 y 8 muestran los impactos del programa en términos de participación laboral mensual para los participantes iniciales y los participantes que acaban ${ }^{15}$. En síntesis, según los resultados obtenidos, podemos afirmar que:

- El programa no tiene efectos en términos de inserción laboral para los jóvenes que lo inician, sin que el análisis desagregado por grupos de edad rebele ninguna diferencia respecto a esta pauta general de impactos nulos.

En cambio, cuando el análisis se realiza considerando únicamente a los participantes que completan todo el programa, aparecen efectos positivos durante casi todos los meses comprendidos entre abril de 2011 y abril de 2013, los cuales no llegan, sin embargo, a contrarrestar el lock-in effect de los primeros meses (los resultados «meses trabajados acumulados» $\mathrm{y}$ «meses trabajados consecutivos» tienen signo positivo, pero el impacto no es estadísticamente significativo).

${ }^{15}$ Véase el detalle de los resultados en los gráficos contenidos en el Anexo B del informe completo. Cabe apuntar que no se han desagregado los resultados según el sexo de los participantes, ya que el $95 \%$ de los jóvenes que han participado en un PCPI-FYAP son varones. 
Gráfico 7: participación laboral mensual (\%), impacto participantes iniciales

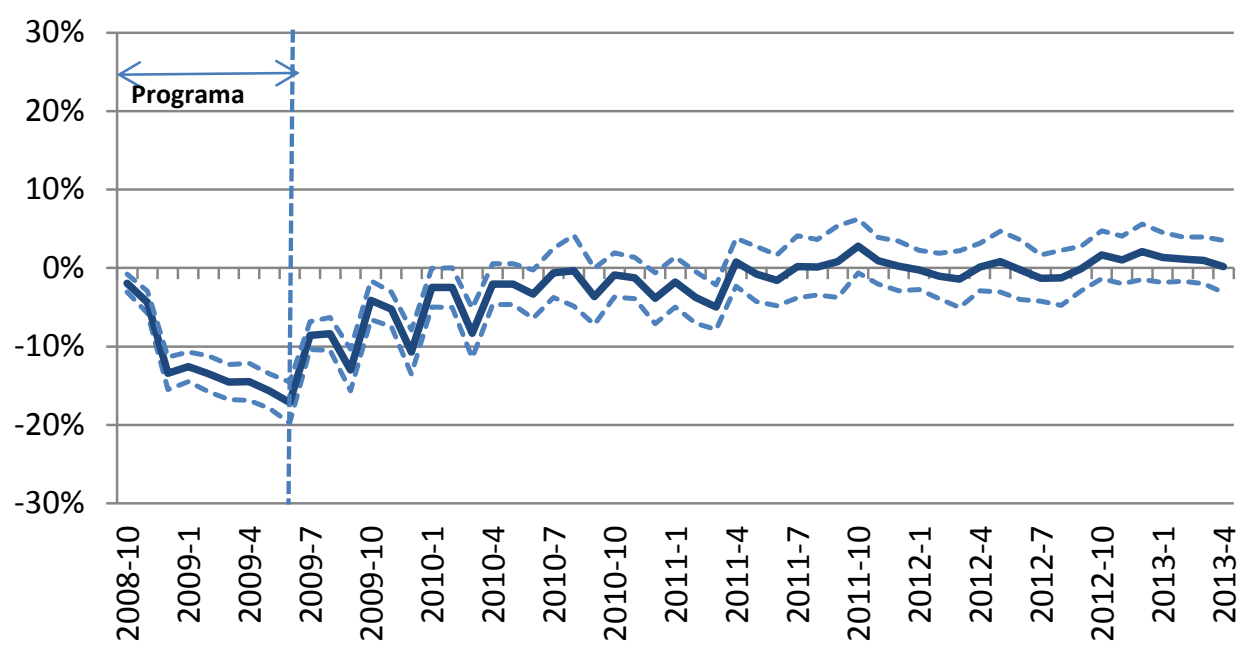

Gráfico 8: participación laboral mensual (\%), impacto participantes que acaban

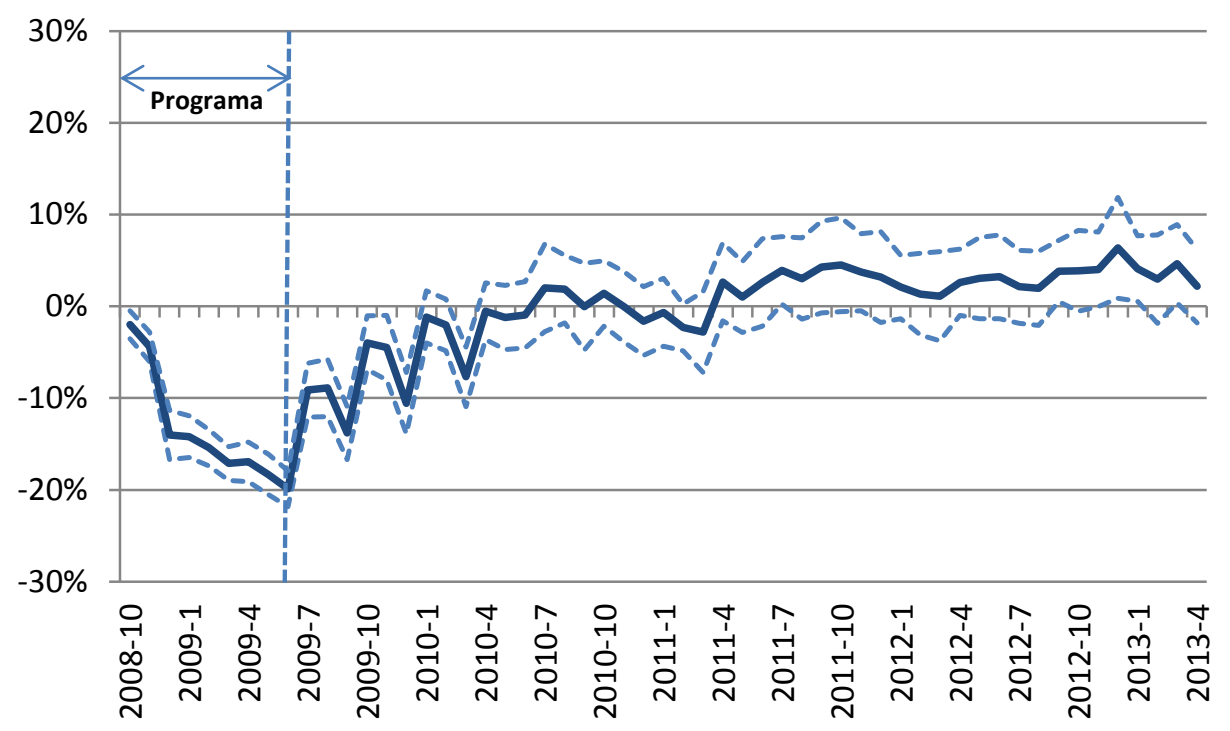




\subsubsection{Impacto educativo}

Los gráficos 9 y 10 contienen la magnitud de los impactos educativos estimados para el conjunto de jóvenes que ingresan al programa y aquellos que lo acaban, respectivamente. A la vista de estos resultados puede afirmarse que:

- El programa tiene efectos positivos sobre los que lo inician tanto en términos de matriculación como de graduación de la ESO, de unas magnitudes superiores a las detectadas en el caso de los PCPI-S. Asimismo, estos impactos se concentran exclusivamente en los jóvenes de entre 16 y 18 años, ya que para los mayores de 18 años los efectos son nulos.

- Por otra parte, en lo que respecta a los CFGM, se repite la misma tendencia observada en los PCPI-S: por una parte, la participación en un FYAP tiene impactos positivos sobre las tasas de matriculación, aunque de una magnitud menor que los PCPI-S; y, por otra parte, estas mayores tasas de matriculación no se traducen en unas mayores tasas de graduación.

Finalmente, cuando nos concentramos en los jóvenes que acaban el programa, la magnitud de los efectos detectados en lo que respecta a la ESO aumentan muy notablemente: así, respecto al grupo de comparación, los participantes de los PCPI-FYAP ven incrementar sus posibilidades de matriculación de la ESO en 10 puntos porcentuales (p.p.) y en 5 p.p. las de obtener el título. No obstante, en lo que respecta a los CFGM, los que terminaron todo el programa consiguen graduarse más que los del grupo de comparación, a pesar del impacto positivo que sí se observa en las matriculaciones. 
Gráfico 9: matriculación y graduación ESO (\% acumulado*): participantes iniciales

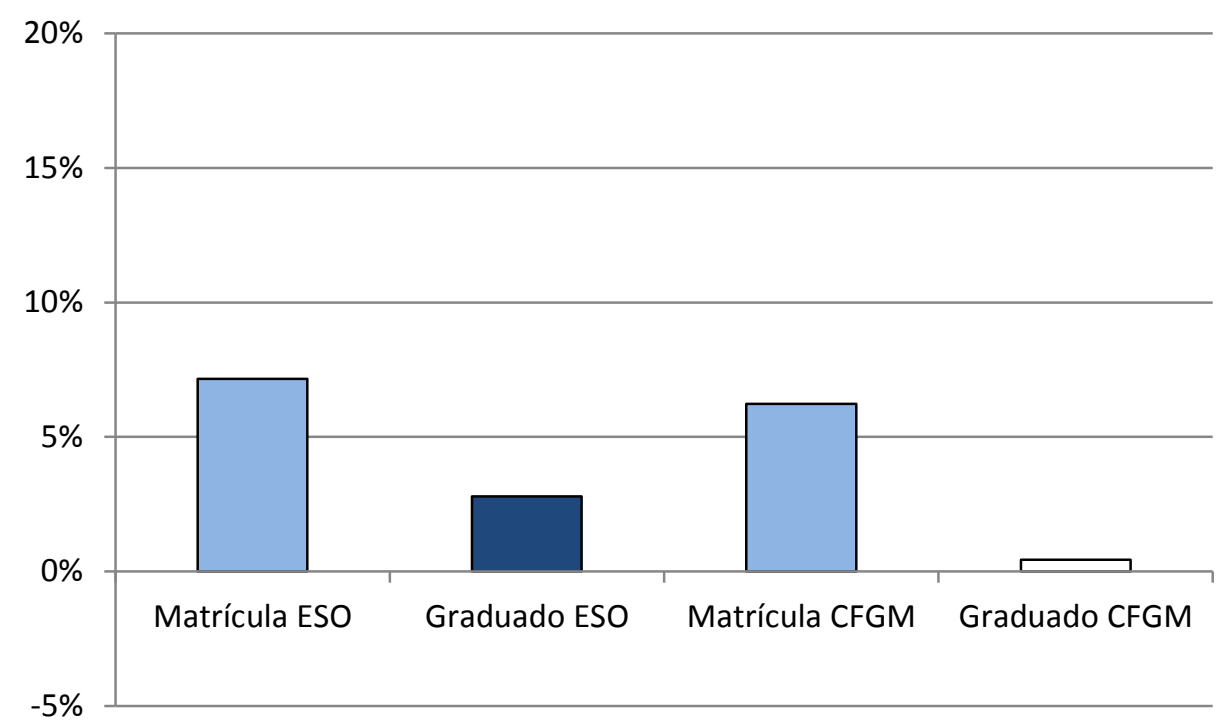

* Cursos 2009-2010 a 2012-2013 (2011-2012, en lo que respecta a la graduación)

Gráfico 10: matriculación y graduación ESO (\% acumulado*), participantes que acaban

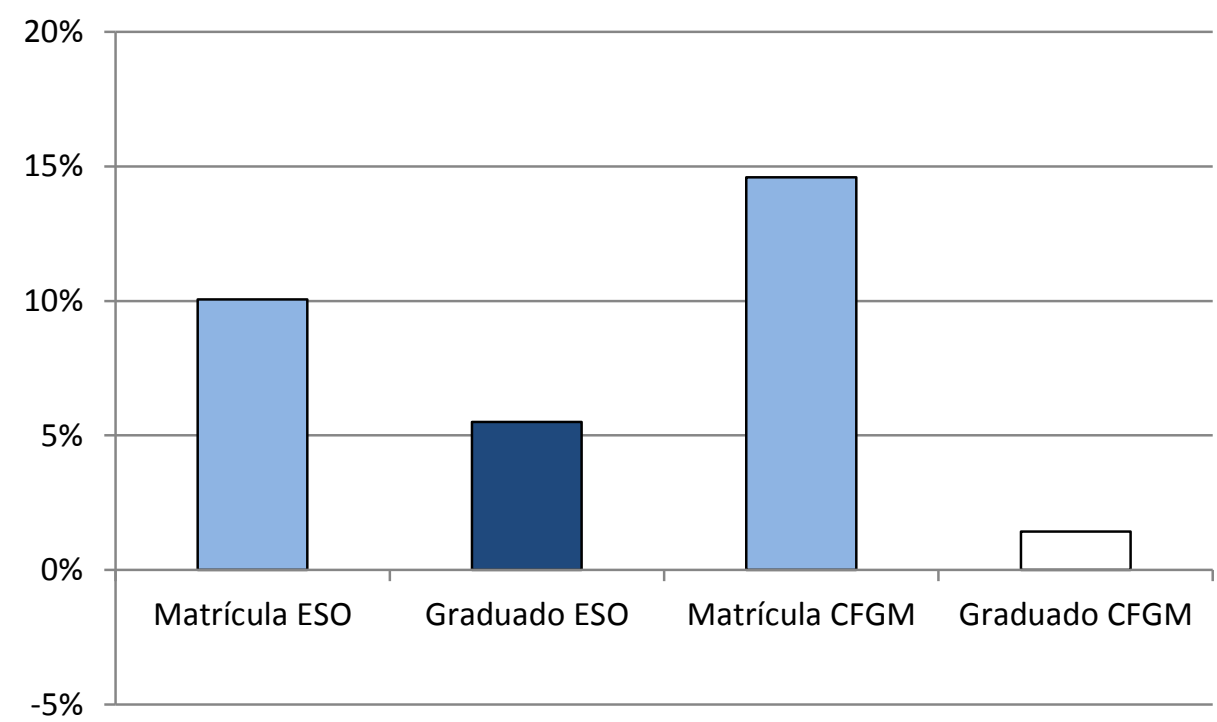

* Cursos 2009-2010 a 2012-2013 (2011-2012, en lo que respecta a la graduación) 


\section{Síntesis e interpretación de los resultados}

Un primer aspecto a destacar es que tanto los PCPI-S como los PCPI-FYAP son efectivos fomentando el retorno al sistema educativo. Así pues, tanto en lo que respecta a la ESO como a los CFGM, los dos programas consiguen impactos positivos en términos de matriculación. Sin embargo, la traslación de esta mayor matriculación en mayores tasas de graduación solamente se produce en el caso de la eso, y de forma más intensa entre los que participan en pcpi-fyap; los impactos son, en cambio, inexistentes en lo que respecta a la obtención de un título en algún cfgm. En definitiva, parece que las medidas de incentivación al retorno educativo que ambos programas ofrecían, como pueda ser el módulo C para la eso o la flexibilización de las pruebas de acceso en el caso de los cfgm, acaban resultando efectivas. Pero en términos de éxito real, una vez desaparecen los incentivos, la mayoría de jóvenes que retoma los estudios no son capaces de graduarse.

En cualquier caso, siguiendo con los resultados educativos, hay que decir que existen diferencias importantes entre los dos programas analizados. Por una parte, los pcpi-fyap parecen tener más éxito a la hora de fomentar el retorno al sistema educativo para completar la eso, mientras que los pcpi-s tienen impactos superiores en lo que respecta a la matriculación en cfgm. Es probable que esta situación tenga que ver con el hecho de que los ies son los encargados de proporcionar los pcpi-fyap, lo cual probablemente asegure un tránsito más fluido hacia la obtención de la eso, mientras que a los centros formativos proveedores de pcpi-s les resulta más natural apostar por los cfgm. Por otra parte, otro aspecto a destacar es la diferencia que existe entre los impactos educativos para los jóvenes que iniciaron el programa y los que efectivamente lo acaban, que es especialmente acusada en el caso de los pcpi-fyap. Así, con este último programa, los impactos sobre las tasas de matriculación y graduación de la eso se duplican cuando consideramos únicamente a los jóvenes que completaron todo el programa. A este respecto, la explicación más plausible podría ser el elevado abandono que se produce en los pcpi-fyap (superior al $40 \%$ ), fruto de un proceso de selección de los participantes que, como veremos en el próximo capítulo, es mucho más laxa que en el caso de los pcpi-s. Por este mismo motivo, en la medida en que los candidatos menos motivados son descartados antes de comenzar el programa, las discrepancias entre los impactos sobre los participantes iniciales y finales son mucho menos acusadas en el caso de los pcpi-s. 
En cuanto a los resultados laborales, los resultados muestran que ambos programas resultan inefectivos cuando consideramos el conjunto de participantes iniciales, pero que, en cambio, aparecen efectos positivos unos dos años después de acabados los programas cuando consideramos únicamente aquellos jóvenes que los completaron íntegramente. Una vez más, esta pauta es más acentuada en el caso de los pcpi-fyap, lo cual corrobora que el proceso de «selección» en el caso de este programa se produce por «abandono», y que los que lo completan ven mejorar tanto sus resultados laborales como educativos.

Finalmente, tanto para los pcpi-s como para los pcpi-fyap, el análisis desagregado16 de los impactos manifiesta que los impactos educativos positivos que se observan se concentran en los jóvenes de entre 16 y 18 años, ya que para el resto de grupos de edad considerados los efectos son nulos o, incluso, negativos. De hecho, en el caso de los pcpi-s, esta pauta también se observa para los resultados laborales, ya que los (modestos) impactos positivos que se detectan solamente se producen entre los participantes más jóvenes. Es probable que la inefectividad de los dos programas entre los jóvenes de más edad tenga que ver con el sesgo de retorno al sistema educativo que impregna ambas intervenciones, un objetivo poco realista cuando se trata de personas que abandonaron las aulas hace quizá demasiado tiempo.

La validez de las estimaciones de impacto obtenidas depende básicamente del grado de similitud que exista entre los participantes de los programas y el conjunto de jóvenes desempleados con los que los hemos comparado. Como ya hemos comentado previamente, la cuestión crucial es que ambos grupos sean idénticos en todo lo que pueda influir sobre los resultados de interés - como pueda ser la inserción laboral-, ya que, de lo contrario, estaremos interpretando como un impacto del programa lo que simplemente es el reflejo de que participantes y no participantes tienen características diferentes (sesgo de selección).

En nuestro caso, dado que las bases de datos disponibles contienen una información muy rica sobre los desempleados (atributos sociodemográficos, experiencia laboral previa, etc.), tenemos motivos para pensar que los impactos estimados mediante la técnica del matching resultan plausibles. No obstante, no todos los factores que pueden influir sobre los resultados de interés (motivación, cargas

\footnotetext{
${ }^{16}$ Por un motivo de espacio, los resultados desagregados no están en este documento pero pueden ser consultados en http://ves.cat/isbV
} 
familiares, etc.) aparecen recogidas en las bases de datos utilizadas, de modo que siempre existe la posibilidad de que participantes y no participantes difieran en estas dimensiones «inobservables». No obstante, lo que sí podemos hacer es simular qué «peso» deberían tener estos factores no observables como para que los impactos detectados mediante la técnica del matching se conviertan en no significativos. En el marco de nuestro estudio, esta magnitud es razonablemente baja, lo cual nos hace confiar en la validez de los resultados obtenidos.17

Otra limitación de los resultados obtenidos tiene que ver con el horizonte temporal sobre el que hemos estimado los impactos. En el caso de los resultados laborales, el periodo de tiempo máximo considerado ha sido de 42 meses después de la finalización de los programas, y de cuatro cursos académicos en el caso de los resultados educativos. No obstante, puede pensarse legítimamente que estas ventanas quizá resulten insuficientes para detectar los efectos de este tipo de programas, y que los impactos resultarían positivos si se dispusiera de un horizonte más amplio. A este respecto, la mejor alternativa pasa por actualizar las estimaciones de impacto de aquí a un cierto tiempo y examinar si los efectos a más largo plazo difieren de los que se han detectado ahora.

\section{Bibliografía}

Arellano, A. (2010). Do training programmes get the unemployed back to work?: A look at the Spanish experience. Revista de Economía Aplicada 28, 39-65.

Becker, S. I M. Caliendo (2007). “mhbounds - Sensitivity Analysis for Average Treatment Effects". IZA Discussion Paper No. 2542, January 2007

Brodaty, T., B. Crépon, and D. Fougère (2001). Using matching estimators to evaluate alternative youth employment programs: evidence from France 1986-1988. In M. F. Pfeifer (Ed.), Econometric evaluation of labour market policies, Volume ZEW Economic Studies, Chapter 13. Physica-Verlag.

\footnotetext{
${ }^{17}$ Los resultados del test estadístico que permite valorar la importancia de un hipotético sesgo de selección por inobservables pueden consultarse en los apartados 3.4 y 3.5 del Anexo A que acompaña al informe.
} 
Caliendo, M., S. Künn and R. Schmidl (2011). Fighting youth unemployment: The effects of active labor market policies. IZA Discussion Paper 6222, Dic 2011.

Card, D., Ibarraran, P., \& Villa, J. (2011). Building and evaluation component for active labour market programs: a practitioner's guide. IZA. Discussion Paper No 6085 , Oct 2011.

Caliendo, M. and S. Kopeinig (2008). Some practical guidance for the implementation of propensity score matching. Journal of Economic Surveys 22, 31-72.

Dehejia, R. H. and S. Wahba (1999). Causal effects in nonexperimental studies: reevaluating the evaluation of training programs. Journal of the American Statistical Association 94, 1053-1062.

Heckman, J. J., R. Lalonde, and J. Smith (1999). The economics and econometrics of active labour market programs. In O. Ashenfelter and D. Card (Eds.), Handbook of Econometrics, Volume 3, Chapter 31, pp. 1865-2097. Elsevier.

Imbens, G. and J. Wooldridge (2009). Recent developments in the econometrics of program evaluation. Journal of Economic Literature 47, 5-86.

Rosenbaum, P. and D. B. Rubin (1983). The central role of the propensity score in observational studies for causal effects. Bioimetrika 70, 41-50.

Rubin, D. B. (1974). Estimating causal effects of treatment in randomized and nonrandomized studies. Journal of Educational Policy 66, 688-701.

Sianesi, B. (2004). An evaluation of the swedish system of active labor market programs in the 1990s. Review of Economics and Statistics 86, 133-155.

Smith, J. and P. Todd (2005). Does matching overcome Lalonde's critique of nonexperimental estimators. Journal of Econometrics 125, 305-353. 